\title{
LA DIFESA DELL'IDENTITÀ DEL CORPO RISORTO CON QUELLO TERRENO NEL DE RESURRECTIONE DI METODIO DI OLIMPO
}

La questione della natura del corpo risorto costituisce senz'altro la problematica centrale del De resurrectione di Metodio di Olimpo $(\dagger 311)^{1}$. L'autore, rispondendo per primo con delle argomentazioni approfondite ed originali ad alcune inquietanti tesi di Origene, si è addentrato in maniera significativa nel dibattito teologico dell'epoca ${ }^{2}$. Tra i personaggi della sua opera, soltanto Aglaofone, che rappresenta una corrente platonica del cristianesimo ${ }^{3}$, non prevede una salvezza delle realtà terrestre. Tutti gli altri tre interlocutori: Eubulio (Metodio), Memiano e Proclo condividono la fondamentale attesa del cristianesimo, cioè quella della risurrezione del corpo terreno, trasformato nel corpo spirituale. Agli inizi del IV sec., tale formula, che univa le due nozioni fondamentali, l'identità e la diversità, era già troppo generica. Nel contesto polemico dell'epoca risultava necessaria la dimostrazione dettagliata del concetto dell'identità corporea. È proprio essa a costituire il punto cruciale del dibattito nel De resurrectione e l'essenziale percorso argomentativo dell'autore.

Lo scopo principale dello studio presente è di identificare dei fattori ritenuti da Metodio necessari e costitutivi del dogma della risurrezione, nonché di analizzare gli argomenti con cui egli cercava di difendere la nozione dell'identità

${ }^{*}$ Dr Mirosław Mejzner SAC - docente alla Cattedra di Teologia Patristica alla Facoltà di Teologia di Università di Cardinal Stefan Wyszynski (UKSW) a Varsavia, e-mail: m.mejzner@uksw.edu.pl.

${ }^{1}$ Methodius, De resurrectione, ed. G.N. Bonwetsch, GCS 27, Leipzig 1917, 219-424, trad. it. M. Mejzner - B. Zorzi: Metodio di Olimpo, La risurrezione, Collana di Testi Patristici 216, Roma 2010.

${ }^{2}$ Cf. J. Becker, La risurrezione dei morti nel cristianesimo primitivo, Studi Biblici 97, Brescia 1991; J. Daniélou, La risurrezione, Torino 1970; G. Dorival, Origène et la résurrection de la chair, in: Origeniana quarta. Die Referate des 4. Internationalen Origeneskongresses, Innsbruck, 2-6 September 1985, ed. L. Lies, Innsbruck - Wien 1987, 291-321; H.E. Lona, Über die Auferstehung des Fleisches. Studien zur frühchristlichen Eschatologie, Berlin - New York 1993; C. Marcheselli-Casale, Risorgeremo, ma come? Resurrezione dei corpi, degli spiriti o dell'uomo? Per un contributo allo studio della speculazione apocalittica in epoca greco-romana: II a.C.-II d.C., Bologna 1988; E. Prinzivalli, La risurrezione nei Padri, in: Dizionario di Spiritualità Biblico-Patristica 45, Roma 2007, 169-288; M. Szram, Ciało zmartwychwstałe w myśli patrystycznej przełomu II i III wieku, Lublin 2010.

${ }^{3}$ Il personaggio di Aglaofone intende la risurrezione come liberazione dell'anima dalla prigionia del corpo e, perciò, l'identifica con la morte fisica dell'uomo, cf. Methodius, De resurrectione I 10, 3. 
del corpo terreno con quello risorto ${ }^{4}$. Si tenterà, inoltre, di specificare le motivazioni fondamentali del suo concetto della risurrezione e della sua posizione ideologica. Verranno esaminati, peraltro, alcuni studi recenti riguardanti la polemica sulla natura del corpo risorto tra Metodio e Origene.

1. L'identità materiale tra il corpo risorto e quello terreno. L'attesa della ricomposizione del corpo materiale nella risurrezione era, agli occhi degli scrittori pagani, il punto più controverso tra le credenze cristiane. Celso la considerava speranza degna semplicemente di vermi ${ }^{5}$. Anche alcuni cristiani di mentalità ellenizzata, influenzati soprattutto dalla tradizione platonica, non condividevano la spiegazione del dogma con le categorie dell'identità fisica ${ }^{6}$

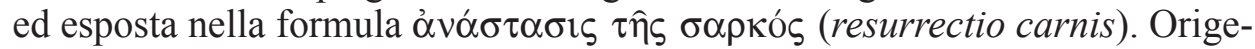
ne, considerandola semplicistica, avanzava la propria teoria della risurrezione

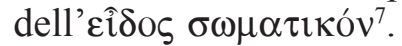

L'obiettivo principale dell'argomentazione di Metodio consisteva nella difesa della formula, diventata ormai una bandiera dell'ortodossia e, quindi, nella spiegazione in maniera organica e razionale della nozione dell'identità materiale tra il corpo terreno e quello risorto. L'identità degli elementi e delle loro qualità fisiche costituiva per lui una condizione necessaria per trattare della soluzione ortodossa. Il fulcro della polemica con Origene non era principalmente di natura dottrinale, ma si focalizzava piuttosto sulla questione filosofica, e cioè sull'individuazione del principio dell'identità corporea. Infatti,

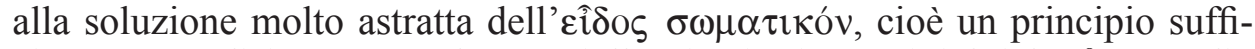
cientemente disincarnato "da non riallacciarsi a immagini fisiche", Metodio ha contrapposto la propria teoria degli elementi costitutivi del corpo con la quale voleva salvaguardare la realtà fisica del corpo risorto9.

a) L'argomentazione biblico-teologica. L'argomentazione di Metodio, adoperata per spiegare la stabilità, la compiutezza e l'identità materiale dell'uomo, è basata su un principio teologico, ricavato principalmente dal passo di Gen 2, 7. Egli sostiene che il diretto coinvolgimento di Dio nella creazione del corpo umano fa di esso un'opera ben ordinata e definita ${ }^{10}$. Perciò tale corpo, individuale e personale di ciascun uomo, è destinato alla risurrezione.

La concezione della ricomposizione del medesimo corpo è contenuta, secondo Metodio, in numerosi passi della Scrittura. Egli era convito che molte

\footnotetext{
${ }^{4}$ Per un'ampia analisi del concetto della risurrezione in Metodio, cf. M. Mejzner, Escatologia di Metodio di Olimpo, SEA 124, Roma - Ząbki 2011, 235-338.

${ }^{5}$ Cf. Origenes, Contra Celsum V 14.

${ }^{6}$ Tale polemica intracristiana è testimoniata anche in altre opere dei primi secoli, cf. A. D'Anna, Studio critico, in: Pseudo-Giustino, Sulla resurrezione. Discorso cristiano del II secolo, Brescia 2001, 142-143, 151-152, 160-161 e 168-177.

${ }^{7}$ Cf. Methodius, De resurrectione I 20-24.

${ }^{8}$ Prinzivalli, La risurrezione nei Padri, p. 222.

${ }^{9} \mathrm{Cf}$. Methodius, De resurrectione II 26-30.

${ }^{10}$ Cf. ibidem II 11, 4-6.
} 
profezie, per esempio quella di ricomposizione di un vaso di creta frantumato $(\text { Ger } 18,3-6)^{11}$, di rialzamento della tenda caduta $(\text { Am 9, 11) })^{12}$, di erezione delle tende per la festa (Lev 23, 39-43) ${ }^{13}$ e, innanzitutto, di vivificazione delle ossa inaridite (Ez 37, 1-14) $)^{14}$, contengono la promessa di Dio che nella risurrezione il corpo sarà ricostruito con gli stessi elementi.

Lo sforzo notevole di salvaguardare la nozione dell'identità tra il corpo terreno e quello risorto emerge anche dalla sua esegesi di 1 Cor $15^{15}$. Nel capitolo, dedicato alla questione della risurrezione, l'Apostolo si è servito tra l'altro dall'esempio del grano e della spiga per illustrare la trasformazione gloriosa del corpo (1Cor 15, 35-38. 42-44). Origene ha sottolineato al massimo la nozione di diversità, evidenziando l'incomparabile splendore del corpo risorto, adombrato nella spiga, rispetto a quello terreno, simboleggiato dal seme gettato in terra. Lo scopo dell'argomentazione di Metodio era proprio contrario, e cioè quello di riassicurare la continuità materiale in ambedue le fasi dell'esistenza corporea ${ }^{16}$. Secondo lui, l'Apostolo si è servito di tale esempio preso dal mondo della natura, soltanto per illustrare in maniera semplice al pubblico maldisposto la verità centrale della sua predicazione, e cioè quella della risurrezione dei corpi morti. Per confermare tale interpretazione Metodio cita 1Cor 15, 36, versetto in cui viene evidenziata sia la necessità della morte che la continuità tra il seme e la spiga. È significativo tuttavia che, nel De resurrectione III 10, egli non riporti né 1 Cor 15,37-38, né altri versetti paolini, da cui emerge piuttosto la nozione della diversità. Inoltre, volendo sminuire una contrapposizione inclusa nel esempio paolino, e cioè quella tra un seme e una spiga, egli ha ampliato la prospettiva, sottolineando la continua ciclicità del processo di vegetazione. In un altro momento, Metodio cercava di dimostrare che la morte non distrugge del tutto il materiale, ma che il germoglio nasce proprio dal seme ${ }^{17}$. Sottolineando la continuità del sostrato materiale, egli era interessato ad assicurare la nozione dell'identità tra il corpo terreno e quello risorto, non negando però una trasformazione avvenuta in quest'ultimo. Secondo lui, il corpo risorto, pur materiale, acquisterà le nuove caratteristiche: immortalità, incorruttibilità, impassibilità, sì da poter essere chiamato spirituale ${ }^{18}$.

b) L'argomentazione cosmologico-antropologica. La precisa visione della ricomposizione del medesimo corpo nella risurrezione è basata su una concezione cosmologica comune nell'antichità. Si intendeva affermare cioè che l'universo è composto dalle quattro sostanze fondamentali ed ogni singolo

${ }^{11}$ Cf. ibidem I 44, 1-2.

${ }^{12} \mathrm{Cf}$. ibidem I 53, 1 .

${ }^{13}$ Cf. ibidem II 21, 1-2.

${ }^{14} \mathrm{Cf}$. ibidem III 9.

${ }^{15}$ Cf. ibidem III 10.

${ }^{16}$ L'esegesi metodiana dell'esempio paolino del grano e della spiga ha influito molto sui posteriori, per esempio sull'esegesi di Didimo il Cieco e quella di Gregorio di Nissa.

${ }^{17} \mathrm{Cf}$. Methodius, De resurrectione I 53, 4.

${ }^{18} \mathrm{Cf}$. ibidem III 16. 
ente materiale è una specifica miscela degli elementi provenienti da esse. Il punto critico della concezione di Metodio consisteva nel provare la possibilità della separazione delle singole particelle da un amalgama di sostanze e, in seguito, della loro ricomposizione nello stesso corpo.

In questa direzione si volge l'argomentazione contenuta soprattutto nel $D e$ resurrectione II 26-30 ed esposta per bocca di un personaggio ortodosso Memiano. Anche se il suo punto di partenza è meramente teologico e si spiega nel principio dell'onnipotenza divina secondo Lc 18, 27: "ciò che è impossibile agli uomini, è possibile a Dio"19, la sua dimostrazione è prevalentemente razionale e basata sugli esempi presi dal mondo della natura. Memiano evidenzia per esempio che gli uomini sanno separare vino dall'acqua, l'argento dal bronzo, il sangue del murice dall'acqua ${ }^{20}$, il rame dall'argento ${ }^{21}$ etc. L'argomento più spettacolare è legato al fenomeno della circolazione dell'acqua nel mondo. Memiano sottolinea che Dio, attraverso il calore del sole agisce sull'acqua salata del mare e distacca da essa l'acqua dolce, portata precedentemente dal fiume, e l'accumula nelle nuvole ${ }^{22}$.

I ragionamenti svolti per dimostrare la possibilità della distinzione dei singoli elementi dalle rispettive sostanze fondamentali avevano per l'autore del De resurrectione lo scopo ben preciso, e cioè render ragionevole l'attesa della ricomposizione del medesimo corpo. Tale interpretazione del dogma cristiano supponeva ovviamente una particolare visione del corpo umano. Metodio sosteneva che esso, nella sua struttura fondamentale, permane identico e personale. Alcuni cambiamenti corporei, verificatisi nell' arco della vita, non negano l'esistenza dell'identità corporea che è garantita dagli elementi costitutivi. Essi formano una base ontologica sulla quale procedono tutte le trasformazioni quantitative. L'insieme degli elementi costitutivi del corpo è stabilito già nell'embrione, formato da Dio nel grembo materno ${ }^{23}$. Da tale impostazione si può dedurre che, secondo Metodio, ciascun elemento costitutivo possiede una caratteristica propria, si potrebbe dire "personale", e perciò è possibile distinguerlo dagli altri. Egli paragonava tale struttura fondamentale ad un alveo dentro cui scorrono delle acque, cioè le sostanze umorali ${ }^{24}$, all'opposizione di Origene che riteneva instabile la sostanza materiale del corpo e la comparava al fiume che continuamente fluisce ${ }^{25}$. Il concetto metodiano dell'identità corporea garantita dagli elementi costitutivi è quindi assai diverso da quello

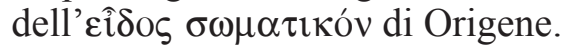

\footnotetext{
${ }^{19} \mathrm{Cf}$. ibidem II 27, 2.

${ }^{20} \mathrm{Cf}$. ibidem II 27, 2. 4.

${ }^{21} \mathrm{Cf}$. ibidem I 59, 1-2.

${ }^{22}$ Cf. ibidem II 28.

${ }^{23} \mathrm{~A}$ favore della propria tesi Metodio adduce un passo di Ger 1, 5, cf. Methodius, De resurrec-

${ }^{24} \mathrm{Cf}$. ibidem II $12,9$.

${ }^{25} \mathrm{Cf}$. ibidem I 22, 3.
} tione II $13,9$. 
Per illustrare la possibilità della ricomposizione del corpo, l'autore del $D e$ resurrectione evidenzia che gli uomini sono capaci di rifare le stesse opere a partire dal medesimo materiale. Anzi, egli sostiene che il processo di passare per una decomposizione verso la ricomposizione è necessario ai fini della purificazione del materiale. Per esempio uno smantellamento del tempio serve a estirpare completamente le radici degli alberi nascosti tra le pietre del muro ${ }^{26}$ oppure la liquefazione del vaso argenteo ad eliminare le impurità del rame ${ }^{27}$. Tali osservazioni servono a Metodio non soltanto a dimostrare la possibilità della risurrezione fisica, ma anche a spiegare il senso terapeutico della morte che riduce il corpo agli elementi costitutivi. A partire da essi Dio onnipotente può compiere il suo progetto originario "come un vasaio che torna a forgiare un vaso per eliminare tutte le deformità e gli schiacciamenti attraverso il rimodellamento, rifacendolo completamente dal principio perfetto ed eccellente" 28 , "a partire dallo stesso materiale originario con cui ciascuno è stato creato" 29 .

c) I motivi teologici e pastorali della composizione del De resurrectione. Le motivazioni per cui Metodio ha intrapreso la difesa del dogma della risurrezione della carne, spiegandolo con le categorie dell'identità materiale tra il corpo risorto e quello terreno, sono sicuramente molto complesse e non sempre chiare. Perciò alcune conclusioni della presente ricerca rimarranno nel campo delle ipotesi.

Un motivo sicuro, e che si presenta in maniera evidente durante la lettura del De resurrectione, è costituito dalla polemica con un'interpretazione innovatrice del dogma, avanzata da Origene, ma considerata pericolosa da Metodio, in quanto troppo contigua alle soluzioni eterodosse. Sicuramente le teorie dell'Alessandrino, autore ecclesiastico di grande fama, urtavano con i dati dottrinali della tradizione asiatica, nonché contro i loro presupposti filosofici, in modo particolare contro l'antropologia unitiva ed il valore positivo della creazione materiale. Metodio, basandosi su questi principi, cercava di dimostrare la ragionevolezza e la congruenza teorica della formula resurrectio carnis e, quindi, la sostenibilità della speranza in essa contenuta.

Alberto D'Anna considera il dibattito presente nel De resurrectione di Metodio successivo a quello svolto nell'omonima opera di Pseudo-Giustino. Lo studioso osserva che nel Commento al Sal 1, 5 di Origene prevalgono già le motivazioni logico-antropologiche della polemica, con un'emblematica obiezione della cosiddetta catena alimentare alla testa. Il problema della separazione degli elementi del corpo nella morte (le motivazioni cosmologico-teologiche)

${ }^{26}$ Cf. ibidem I 41.

${ }^{27}$ Cf. ibidem I 43, 2; 59, 2.

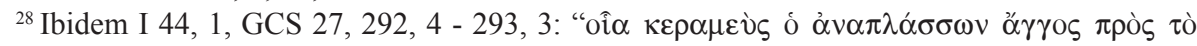

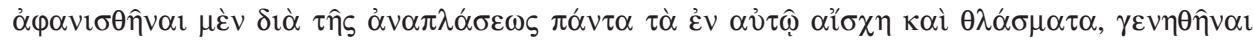

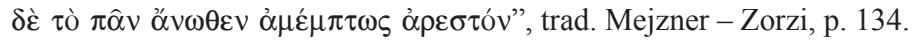

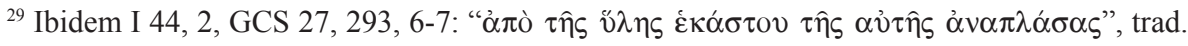
Mejzner-Zorzi, p. 135. 
appare superato, perché vengono affrontate alcune aporie dovute a possibili eventi successivi alla decomposizione ${ }^{30}$. Tanto più risultano superate le motivazioni pratiche delle obiezioni contro la risurrezione della carne che stanno, in qualche maniera, alla base della ferocia della persecuzione dei cristiani avvenuta nel 177 a Lione e a Vienne, in Gallia meridionale ${ }^{31}$.

La classificazione delle motivazioni, proposta da D'Anna, dimostra in modo chiaro ed approfondito l'evoluzione argomentativa e lo sviluppo della polemica intorno alla risurrezione a livello teoretico. C'è, tuttavia, un'altra motivazione, che si potrebbe chiamare esistenziale, e che assume maggior rilievo particolarmente al tempo delle persecuzioni, spingendo gli autori cristiani (e predicatori) ad indicare esplicitamente la carne come l'oggetto della risurrezione e a proclamare con forza il realismo di tale speranza.

È sintomatico che nel tempo delle persecuzioni crescesse una tensione apocalittica, manifestatasi in diverse forme, ma il cui denominatore comune era costituito sempre dall'attesa dell'intervento divino che avrebbe posto fine all'intollerabile ordine attuale e che avrebbe giustamente ricompensato le tribolazioni dei fedeli nel suo regno. In questo senso anche l'età dioclezianea non solo favoriva delle speculazioni sulla morte e sulla sorte ultima dell'uomo, ma rendeva necessarie delle spiegazioni sul senso delle sofferenze, diventate una prova realmente vicina ${ }^{32}$.

Un'analisi della dimensione esistenziale e psicologica delle riflessioni escatologiche di un autore, in questo caso di Metodio, è rischiosa in una sede storica. Tuttavia, essa sembra opportuna, prendendo in considerazione che egli non era un mero pensatore, ma verosimilmente anche un vescovo. La cura pastorale dei fedeli era per lui di primaria importanza. Metodio ha scritto il De resurrectione in una particolare situazione politica e sociale che influiva sul modo di pensare, di sentire e di sperare dei cristiani. La composizione dell'opera non è causata soltanto da fattori teoretici, dalla necessità di confutare le obiezioni dei filosofi pagani e degli gnostici o di opporsi ad alcune tesi origeniane, ma probabilmente anche dall'urgenza di confermare la fede nella risurrezione del corpo, esposto alle tribolazioni ed al martirio.

Una traccia sicura e commovente della presenza della motivazione esistenziale nel De resurrectione è ricavabile dall'esegesi metodiana di Sal 65, 10-12 $2^{33}$. Dopo aver combattuto l'esegesi avversaria, l'autore afferma che nelle prove menzionate nel passo biblico sono adombrate le sofferenze dei

${ }^{30}$ Cf. D’Anna, Studio critico, p. 131-140, innanzitutto n. 33.

${ }^{31}$ La lettera (forse di Ireneo), inviata dalla comunità di Lione a quella di Smirne, per raccontare l'azione persecutoria, testimonia la percezione, da parte pagana, della credenza nella risurrezione come fondante e caratterizzante la fede dei cristiani. L'annientamento dei cadaveri (oltraggiati ed esposti al pubblico per sei giorni, poi bruciati e ridotti in polvere dispersa nel fiume Rodano) volle essere una sorta di dimostrazione populista dell'assurdità di tale fede, cf. Martyrium Lugdunensium LXIII.

32 Un'analisi dell'influenza delle persecuzioni sull'escatologia cristiana, cf. M. Wysocki, Eschatologia okresu prześladowań na podstawie pism Tertuliana i Cypriana, Lublin 2010, 251-266.

${ }^{33} \mathrm{Cf}$. Methodius, De resurrectione I 54-56. 
patriarchi, profeti, apostoli e martiri cristiani. Al termine di questa esposizione, Metodio, rompendo la distanza narrativa, si autoinserisce in questa catena dei testimoni privilegiati della fede, augurandosi di poter passare vittoriosamente per la prova ${ }^{34}$. Questo insolito atteggiamento dell'autore riflette non soltanto un'atmosfera di pericolo, ma dimostra un forte legame intrinseco, sentito da lui, tra il martirio e la fede nella risurrezione della carne.

Alla luce di queste considerazioni si può avanzare un'ipotesi. Sembra molto probabile che la difesa della risurrezione della carne intrapresa da Metodio contro le interpretazioni spiritualizzanti sia stata spinta, oltre alle ragioni dottrinali, anche dalle profonde motivazioni esistenziali e pastorali. Convinto che la professione del dogma, nella sua interpretazione tradizionale, costituisca un incoraggiamento dei fedeli esposti alle tribolazioni, egli combatte la concezione origeniana della risurrezione, unita a quella dell'apocatastasi, temendo che essa possa raffreddare il desiderio del martirio e, di conseguenza, provocare nelle comunità cristiane un comportamento meno radicale di fronte alle persecuzioni.

Questa ipotesi, come tante altre, è destinata a non trovare una conferma probante, perché Metodio non esprime direttamente tale opinione ${ }^{35}$. Una certa luce su questo dilemma si potrebbe, tuttavia, ricavare dagli Atta Phileae ${ }^{36}$, del vescovo di Tmuis, martirizzato nel 306. Durante il suo confronto con Culciano che dichiara di preoccuparsi della vita ( $\psi v \chi \eta ́)$ di Filea, il vescovo incolpato introduce volontariamente la questione della risurrezione della carne e ribadisce che la salvezza riguarda non solo l'anima, ma anche il corpo. In questo contesto è significativa un'affermazione di Culciano, trasmessa dal Papiro Bodmer $X X^{37}$, che accusa Filea di aver causato, con la sua ostinazione, la morte di molti, a differenza di un certo Pierio che sottomettendosi al suo comando, ha salvato tanti ${ }^{38}$. Non addentrandoci nelle speculazioni sul tipo di sottomissione dell'ecclesiastico menzionato ${ }^{39}$, si osservi la percezione di Pierio, sostenitore dell'apocatastasi ${ }^{40}$, quale contraltare negativo dell'inflessibile Filea di Tmuis, da parte degli avversari della linea origeniana. Circolava, quindi, almeno in alcuni ambienti, un sospetto, se non la convinzione, che le idee escatologiche di Origene si traducessero più facilmente in un comportamento meno eroico.

${ }^{34} \mathrm{Cf}$. ibidem I 56, 9.

${ }^{35}$ Dal trattato De martyribus di Metodio sono rimasti soltanto due frasi (ed. G.N. Bonwetsch, GCS 27, 520).

${ }^{36}$ Acta Phileae, ed. A. Bastiaensen, in: Atti e passioni dei martiri, Milano 1987, 280-337.

${ }^{37}$ Per l'analisi delle fonti agiografiche su Filea di Tmuis, cf. G. Kortekaas, Prefazione: Atti di Filea, in: Atti e passioni dei martiri, p. 249-270.

${ }^{38}$ Cf. Acta Phileae V-VI (Papyrus Bodmer XX), ed. Bastiaensen, p. 318-323.

${ }^{39}$ Per una breve descrizione del problema, cf. E. Prinzivalli, Magister Ecclesiae. Il dibattito su Origene fra III e IV secolo, SEA 82, Roma 2002, 80-82. Nonostante qualche offuscamento della fama postuma di Pierio, probabilmente morto martire insieme al fratello Isidoro, è stata dedicata a lui una chiesa ad Alessandria, cf. R. Lorenz, Eine Pierius-Memoria in Alexandrien, ZKG 99 (1988) 87-92.

${ }^{40}$ A tale opinione spinge la notizia di Photius, Bibliotheca 119 , che attesta che Piero affermava la preesistenza delle anime. 
Anche Metodio condivideva tale sospetto? La risposta a tale domanda deve rimanere ipotetica. È sicuro, comunque, che egli combatte non solo la concezione spiritualista della risurrezione, ma anche la teoria dell'apocatastasi ${ }^{41}$. Secondo lui, il giudizio finale svolto sull'uomo nella sua interezza (corpo e anima) avrà un duplice risultato - la salvezza o la condanna - corrispondente alle opere compiute da ciascuno. Infatti, la motivazione escatologica è fondamentale nella comprensione della sua impostazione etica. Egli è profondamente convinto che la risurrezione dell'uomo per la vita eterna sia una tappa decisiva dell'esistenza umana, in cui la carne, essendo stata provata e purificata attraverso le sofferenze coraggiosamente sopportate fino alla morte, viene resa incorruttibile e immortale per poter partecipare, anche essa, alle glorie del paradiso.

La difesa del realismo della risurrezione è lo scopo principale dell'opera di Metodio. Costretto a rispondere alle accuse degli ambienti platonizzanti e ad opporsi alla soluzione proposta da Origene, egli ribadisce l'interpretazione tradizionale del dogma, avanzando una visione precisa del corpo e della sua risurrezione. Gli elementi costitutivi del corpo, cioè il sostrato materiale che lo edificava fin dall'inizio, appartengono all'identità dell'individuo e, perciò, nella risurrezione saranno riuniti da Dio nella medesima forma. Grazie a tale visione, il pericolo delle tribolazioni può essere affrontato con fermezza, perché non a un altro, ma proprio a questo corpo materiale spetta risorgere.

2. L'identità della forma umana. L'assicurazione dell'identità della forma del corpo umano nella risurrezione costituisce per Metodio un obiettivo di non minore importanza di quello precedente. In realtà ambedue i fattori, e cioè l'identità degli elementi materiali e dell'assetto delle membra, sono indispensabili, secondo lui, per sostenere l'identità corporea. Il focalizzarsi dell'autore su tale problematica è un effetto diretto della sua polemica con l'idea

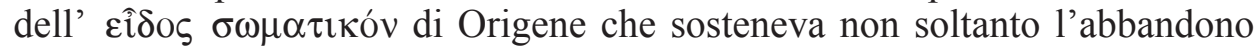
della materia terrena nella risurrezione, ma anche una radicale modificazione dell'assetto delle membra, dovuto all'adattamento del corpo risorto al regno dei cieli.

a) L'argomentazione biblico-teologica. Una delle categorie antropologiche, preferite da Metodio, era quella biblica dell'uomo fatto ad immagine e somiglianza di Dio (Gen 1, 26). Nel De resurrectione I 34-35, egli espone in maniera molto densa il proprio concetto della creazione dell'uomo, sottolineando il diretto coinvolgimento di Dio che ha plasmato uomo dandogli forma

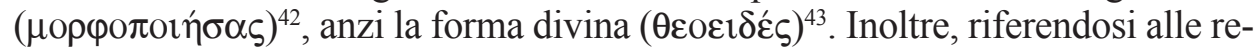
gole dalla vita politico-sociale, egli sottolinea che l'importanza di un'immagine del re non deriva dal tipo del materiale utilizzato per la produzione, ma consiste

\footnotetext{
${ }^{41}$ Cf. Methodius, De resurrectione III 21, 8-9.

${ }^{42}$ Ibidem I 35, 4, GCS 27, 275, 9. Nel versetto di Gen 1, 26 nei LXX sta il verbo $\pi$ oté $\omega$.

${ }^{43}$ Ibidem I 35, 2, GCS 27, 274, 4.
} 
nella somiglianza al sovrano, cioè nell'aspetto formale. Similmente, la dignità dell'uomo e la sua immortalità provengono dall'essere l'immagine di $\mathrm{Dio}^{44}$.

Il famoso passo di Ger 18, 3-6, contenente l'immagine dell'orciaio che con le mani forma i vasi di creta, è stato utilizzato da Metodio coma un'illustrazione della futura risurrezione e, in particolare, della ricomposizione del corpo dai medesimi elementi costitutivi e nella stessa forma. Tale scopo era anche del suo discorso sulla necessità della fusione di una danneggiata statua d'oro, ai fini di ripristinare la sua bellezza originaria ${ }^{45}$. Paragonando l'uomo insidiato dal peccato a tale statua corrotta, egli spiega che la perfezione della forma originaria, espressa nella simmetria delle membra, non può essere rifatta con una semplice riparazione, ma solo attraverso la fusione del materiale ed un nuovo modellamento.

"Perciò anche il Logos discese ad abitare in mezzo a noi e s'incarnò prendendo il nostro corpo, affinché avendo restaurato l'immagine in modo più conveniente a Dio, poiché era da lungo tempo corrotta, la facesse risorgere in modo indistruttibile"46.

Metodio trova un fondamento solido della sua tesi circa l'identità della forma del corpo risorto a quella terrena dell'uomo soprattutto nel campo cristologico. Secondo lui, sia l'evento della trasfigurazione di Gesù che la sua risurrezione dimostrano la persistenza della forma umana nel processo di glorificazione del $\operatorname{corpo}^{47}$. Peraltro, il fatto che il Logos divino assunse tale forma rivela il suo carattere unico e privilegiato ${ }^{48}$. Nel mondo corporeo delle creature

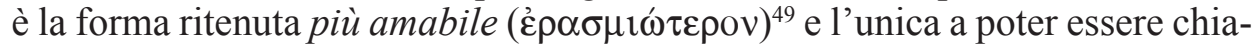
mata divina $\left(\theta \varepsilon 0 \varepsilon 1 \delta \varepsilon \varepsilon_{\zeta}\right)^{50}$. Per Metodio risulta logico che tutti gli esseri creati ad immagine di Dio e, quindi, non solo gli uomini e le loro anime, ma anche gli angeli nei loro corpi intelligibili, debbano possedere tale forma ${ }^{51}$.

Metodio trova un argomento in favore della persistenza dell'assetto delle membra anche nel campo dell'escatologia intermedia. Egli sostiene che sia il

${ }^{44}$ Cf. ibidem II 24.

${ }^{45} \mathrm{Cf}$. ibidem I 43, 5 - 44, 3. Il contesto escatologico è sottolineato maggiormente grazie al collegamento di Ger 18, 3-6 a Rom 9, 21 e Dan 12, 2-3.

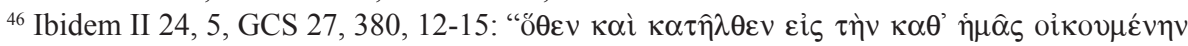

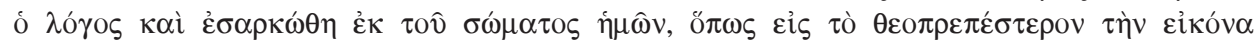

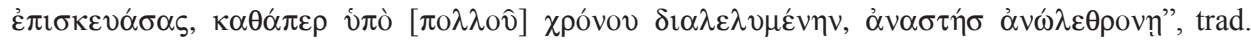
Mejzner - Zorzi, p. 226.

${ }^{47}$ Cf. ibidem III 12-14.

${ }^{48}$ Cf. ibidem II 18, 8; 24, 5. La motivazione teologica dei cristiani si lega perfettamente all'opinione popolare degli antichi che consideravano la struttura corporea dell'uomo come la realtà più armoniosa esistente in natura e dotata di un significato quasi simbolico.

${ }^{49} \mathrm{Cf}$. ibidem III 15, 1, GCS 27, 411, 22.

${ }^{50} \mathrm{Cf}$. ibidem I 35, 2, GCS 27, 274, 4.

${ }^{51}$ Ibidem III 15, si oppone alla tesi riguardante le trasformazioni dell'assetto delle membra nella risurrezione, anche attraverso le domande retoriche circa le eventuali forme alternative (poligonale, cubica o piramidale) che potrebbero riflettere le speculazioni post-origeniane. 
fenomeno delle apparizioni dei morti che la parabola del ricco epulone e di Lazzaro (Lc 16, 19-31) dimostrano la natura corporea dell'anima ${ }^{52}$ che, pur separata dalla carne, conserva la medesima forma. Se è cosi, anche il corpo risorto avrà il medesimo assetto delle membra del corpo terreno, perché gli elementi costitutivi saranno riuniti all'anima, adattandosi alla sua forma.

Un campo di importanza particolare per la valorizzazione degli argomenti è quello esegetico. Metodio accusa Origene di non saper discernere i passi da interpretare letteralmente da quelli che esigono un'allegoresi ${ }^{53}$. Secondo lui il versetto di Mt 8, 12b: vi sarà pianto e stridore di denti, interpretato allegoricamente dall'Alessandrino ${ }^{54}$, deve essere inteso alla lettera ${ }^{55}$. Questo passo, diversamente da quello di Lc 16, 19-31 che narrava delle condizioni delle anime nell' interim $^{56}$, si riferisce alle pene dei dannati nella Geenna, e cioè al periodo dopo la risurrezione ${ }^{57}$. L'autore del De resurrectione, rievocando inoltre l'esempio della trasfigurazione, sottolinea la presenza delle membra nel corpo risorto. Secondo lui, Origene si contraddice affermando una volta la risurrezione dell' $\varepsilon \hat{i} \delta o \varsigma$ e, quindi - secondo la comprensione metodiana - della forma umana, e poi negando la presenza delle membra ${ }^{58}$.

Henri Crouzel, che non perde occasione per sottolineare il malinteso della concezione dell'Eî́os da parte di Metodio, includendolo, peraltro, tra il numero degli "Anthropomorphites contre lesquels Origène a lutté toute sa vie", ammette, tuttavia, che egli ha percepito e criticato un punto debole della dottrina dell'Alessandrino, e cioè 'l'absence dans le corps ressuscité des membres nécessaires pour le monde de génération et de devenir"59. Tale idea origeniana è espressa nel De resurrectione anche dal personaggio di Aglaofone che, interpretando spiritualmente il versetto di Mt 22, 30: "alla risurrezione infatti non si prende né moglie né marito, ma si è come angeli nel cielo", afferma l'assenza

${ }^{52} \mathrm{Cf}$. ibidem III 18. Per Metodio la forma è una qualità del corpo che non potrebbe essere disgiunta da esso e data all'anima, cf. ibidem III 6, 10. Per un esame più approfondito, cf. M. Mejzner, L'eidos e l'ochèma. La critica al concetto origeniano di risurrezione nel contesto dell'escatologia intermedia nel "De resurrectione" di Metodio di Olimpo, in: Origeniana Decima: Origen as Writer. Papers of the $10^{\text {th }}$ International Origen Congress, Krakow: 31.08 - 04.09.2009, ed. S. Kaczmarek - H. Pietras, Bibliotheca Ephemeridum Theologicarum Lovaniensium 244, Leuven - Paris - Walpole 2011, 907-918.

${ }^{53} \mathrm{Cf}$. Methodius, De resurrectione III 8, 3. In seguito Metodio indica altri due brani (Sal 21, 19 e Lc 3,11$)$ da intendere letteralmente che Origene ha inteso in maniera allegorica. Per un esame più approfondito, cf. E. Prinzivalli, L'esegesi biblica di Metodio di Olimpo, SEA 21, Roma 1985, 45-47.

${ }^{54} \mathrm{Cf}$. Methodius, De resurrectione I 24, 1.

${ }^{55} \mathrm{Cf}$. ibidem III 7-8.

${ }^{56}$ Origene sottolinea, infatti, che la parabola del ricco epulone e di Lazzaro non è da riferire al tempo della risurrezione, cf. Methodius, De resurrectione III 17, 2.

${ }^{57}$ Sul piano dottrinale, l'idea delle pene infernali cominciava ad essere rievocata come una conferma della risurrezione fisica, per esempio cf. Tertullianus, De resurrectione mortuorum 35.

${ }^{58} \mathrm{Cf}$. Methodius, De resurrectione $\mathrm{III}$ 7, 3; 7, 9 .

${ }^{59} \mathrm{Cf}$. H. Crouzel, Les critiques adressées par Méthode d'Olympe et ses contemporains à la doctrine origenienne du corps ressuscité, "Gregorianum" 53 (1972) 712. 
della carne nel mondo dei risorti. Secondo lui, la risurrezione corporea nelle stesse membra e nella stessa forma, implicherebbe la permanenza di tutti i bisogni terreni, procreazione inclusa, il che viene contraddetto dal versetto ${ }^{60}$.

Le più importanti riflessioni di Metodio legate all'esegesi di Mt 22, 30 sono contenute nel De resurrectione I 48, 4 - 51, 4. L'autore sostiene la mancanza della procreazione nel mondo dei risorti, il che non significa la mancanza delle membra e, quindi, il cambiamento della forma umana. Tuttavia, egli non si esprime chiaramente sull'utilità di tale membra nel paradiso escatologico. Collocando, tuttavia, tale luogo sulla terra, egli dimostra indirettamente l'adeguatezza dell'ubicazione dei corpi formati come nella prima creazione.

b) Un difficile passo del Symposium. In un passo del Symposium, dialogo in cui le istanze dogmatiche riguardanti l'escatologia non sono ancora esposte in termini così precisi che nell'opera dedicata essenzialmente a tale problematica, Metodio sembra ipotizzare qualche trasformazione del corpo, successiva alla risurrezione. Per bocca di Tisiana, egli esprime l'opinione che, terminato il millennio, il passaggio dalla terra rinnovata alla sede superiore nei cieli si rispecchierà anche nella condizione del corpo che:

"si muterà dall' aspetto umano e corruttibile in grandezza e bellezza angelica" ${ }^{\prime \prime 1}$.

Le espressioni usate nel passo del Symposium suonano senz'altro diverse da quelle esposte nel De resurrectione, suscitando alcuni interrogativi ${ }^{62}$. Infatti, in questa ultima opera non appare la questione del millenarismo, il paradiso terrestre è ritenuto la sede eterna dei salvati e la distinzione ontologica tra gli uomini e gli angeli è molto netta. Peraltro, il corpo risorto è ritenuto perfetto (incorruttibile, immortale, impassibile, abitato dallo Spirito Santo) e la sua forma è chiamata divina e più amabile. Sembra che Metodio, spinto da maggiori esigenze dottrinali, effettui delle precise correzioni di alcune idee esposte nel Symposium.

La differenza di prospettiva nelle due opere è facilmente percepibile dal divergente approccio esegetico a Lev 23, 39-43. Metodio, nel De resurrectione II 21 si limita ad interpretare la festa delle tende come la risurrezione del corpo, la cui composizione materiale viene fortemente sottolineata. Le questioni

${ }^{60} \mathrm{Cf}$. Methodius, De resurrectione I 7, 2. Origene, nell'esegesi di Gen 3, 21 (Theodoretus Cyrensis, Quaestiones in Genesim 39, PG 80, 140), ha avanzato l'obiezione circa l'utilità delle membra già nel paradiso protologico, cf. M. Simonetti, Alcune osservazioni sull'interpretazione origeniana di Gn 2,7 e 3,21, “Aevum” 36 (1962) 370-381.

${ }^{61}$ Methodius, Symposium IX 5, 255, ed. H.A. Musurillo - V.-H. Debidour, SCh 95, Paris

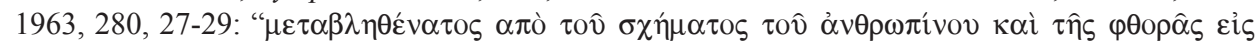

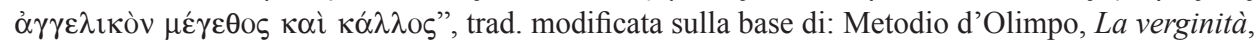
ed. N. Antoniono, Collana di Testi Patristici 152, Roma 2000, 149.

${ }^{62}$ Per esempio G.N. Bonwetsch (Die Theologie des Methodius von Olympus, Abhandlungen der Königlichen Gesellschaft der Wissenschaften zu Gottingen, Philologisch-Historische Klasse, Neue Folge Band VII/1, Berlin 1903, 125) si domanda se le espressioni contenute nel Symposium IX 5 non siano in contraddizione con la tesi esposta da Metodio nel De resurrectione I 51, e cioè che gli uomini diventeranno come angeli, ma non angeli. 
della durata di tale festa e della successiva dinamica escatologica sono coperte dal silenzio ${ }^{63}$.

Nel Symposium IX, l'esegesi allegorica del passo è molto più ampia e focalizzata su numerosi particolari ${ }^{64}$. Innanzitutto Metodio allarga una prospettiva cronologica, soffermandosi non soltanto sul significato delle tende, ma cercando anche di spiegare cosa significa il passaggio da esse alla terra promessa. Egli situa, quindi, Lev 23, 39-43 nel contesto dell'intero esodo, dalla fuga dall'Egitto fino al compimento della promessa, dando rilievo all'importanza del periodo desertico nelle tende, ma affermando anche il suo carattere provvisorio e temporaneo. Tale impostazione è dovuta sicuramente a vari fattori: da una parte Metodio delinea il quadro completo dei tempi escatologici, ivi incluso il millennio intermedio, dall'altra egli sviluppa il motivo della marcia degli Israeliti verso la meta finale in conformità all'idea essenziale dell'opera, e cioè quella della progressiva ascesa dell'uomo verso Dio ${ }^{65}$. Ciononostante egli è molto attento a salvaguardare il dato dottrinale della risurrezione della carne e della persistenza del corpo non solo nel paradiso, ma anche nei cieli ${ }^{66}$. I dettagli della sua esegesi rivelano con chiarezza come tale aspetto sia al centro dei suoi interessi.

Il punto critico dello scenario delineato è costituito dal carattere temporaneo e delimitato del soggiorno degli Israeliti nelle tende, identificate da Metodio con i corpi. Secondo i criteri della logica, l'abbandono di tale abitazione provvisoria per conquistare la terra promessa, dovrebbe significare nella sua allegoresi il distacco delle anime dai corpi risorti. È proprio a questo punto che egli interviene con una spiegazione particolare e benvoluta: la fine della sosta nelle tende significa il passaggio verso i cieli ed implica il mutamento $(\mu \varepsilon \tau \alpha \beta \lambda \eta \theta \dot{\varepsilon} v \alpha \tau \sigma \varsigma)^{67}$ delle tende, ma non il loro abbandono ${ }^{68}$. Questa trasformazione consisterà nel passaggio "dall' aspetto umano e corruttibile in grandezza e bellezza angelica" ${ }^{69}$.

Da una parte il concetto di tale mutamento è perfettamente conforme ai dati dottrinali della tradizione di Metodio, dall'altra è consono al linguaggio del

${ }^{63}$ Per un esame più approfondito, cf. M. Mejzner, Methodius: millenarist or anti-millenarist?, in: Methodius of Olympus. State of the art and new perspectives, ed. K. Bracht, TU, Berlin 2017 [in via di pubblicazione].

${ }^{64}$ Metodio, allegorizzando il testo, tende a dare una spiegazione al maggior numero possibile di elementi. È un procedimento tipicamente origeniano.

${ }^{65}$ Questo ultimo motivo riflette le idee predilette di Origene (In Exodum hom. 9, 4; In Numeros hom. 17, 4).

${ }^{66}$ L'intenzione metodiana di salvaguardare il dato della persistenza corporea per tutta l'eternità è giustamente sottolineata da L.G. Patterson (Methodius 'Millenarianism, StPatr 24:1993, 312 n. 9-10).

${ }^{67}$ Methodius, Symposium IX 5, 255, SCh 95, 280, 27-28.

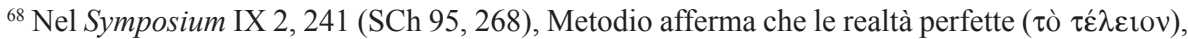
che saranno compiute alla seconda venuta del Signore, sono "il regno dei cieli e la risurrezione"

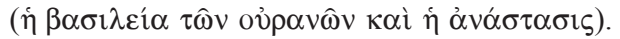

${ }^{69}$ Ibidem IX 5, 255, SCh 95, 280, 27-29. 
Symposium, dove l'elevazione ai cieli e l'ideale della somiglianza alla vita angelica sono fortemente enfatizzati. Sostenuto il mutamento dell'aspetto corporeo, l'autore evita ogni accenno alla sostanza o al sostrato materiale. Tanto più egli non afferma che gli uomini diventano gli angeli, ma dichiara solo che ne assumono alcune qualità ${ }^{70}$. L'espressione usata Symposium IX 5, pur particolare, non sembra sostanzialmente contraddire quanto sarà detto nel De resurrectione $e^{71}$.

Infatti, anche in quest'ultima opera, Metodio parla del mutamento di $\sigma \chi \hat{\eta} \mu \alpha$ del cosmo e del corpo ${ }^{72}$. Anzi, egli utilizza il verificarsi di tale trasformazione, che si manifesta "nel mutamento della figura del bambino nell'uomo perfetto, quando il bambino cresce in bellezza e altezza"

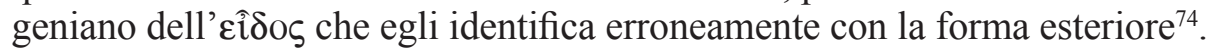

Metodio, scrivendo il De resurrectione, era preoccupato di difendere la formula dogmatica e, come sembra, si è reso conto delle imprecisioni, esposte nell'opera precedente, imprecisioni che avrebbero potuto essere usate dai suoi critici. Perciò, la sua esegesi di Lev 23, 39-43, in quest'ultima opera,

${ }^{70}$ La persistenza della materia dopo il millennio è sostenuta anche da Ireneaus, Adversus haereses V 36, 1, ed. A. Rousseau - L. Doutreleau, SCh 153, Paris 1969, 452-454, trad. it. E. Bellini, in: Ireneo di Lione, Contro le eresie e gli altri scritti, Milano 1997, 480-481: "Ed è giusto, perché, essendo reali gli uomini, reale deve essere anche il loro trasferimento; non vanno però verso il nulla, ma progrediscono nell'essere. Infatti non è annientata né la sostanza né la materia della creazione - perché verace è colui che l'ha stabilita -, ma passa la figura di questo mondo (1Cor 7, 31), cioè gli elementi nei quali è avvenuta la trasgressione, perché l'uomo è invecchiato in essi". Un passo più deciso fa, per esempio, Tertulliano che vedeva i salvati, dopo il millennio "demutati in atomo in angelicam substantiam" (Adversus Marcionem III 24, 6, ed. R. Braun, SCh 399, Paris 1994, 206, 41). La trasformazione angelica dell'uomo dopo il millennio è una nozione che Metodio probabilmente abbia ripreso dalla tradizione, cf. anche Iustinus, Dialogus cum Tryphone 81, 4.

${ }^{71}$ Di tale opinione è, per esempio, A. Biamonti, L'escatologia di Metodio d'Olimpo, "Rivista Trimestrale di Studi Filosofici e Religiosi" 4 (1923) 198: "Metodio afferma soltanto che noi prenderemo la grandezza e la forma degli angeli, ma non dice che diventeremo angeli; tuttavia bisogna riconoscere che tra il De res. e il Symp. si nota a questo proposito una certa differenza, dovuta soprattutto all'intento diverso delle due opere (il Symp. si propone l'elogio della castità, il De res. difende la risurrezione della carne)". Anche E. Prinzivalli (Magister Ecclesiae, p. 160 n. 49) sottolinea l'essenziale accordo tra le due impostazioni.

${ }^{72} \mathrm{Cf}$. Methodius, De resurrectione I 48, 1-2.

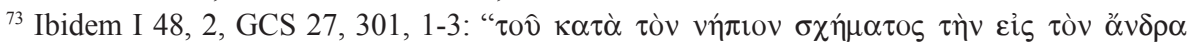

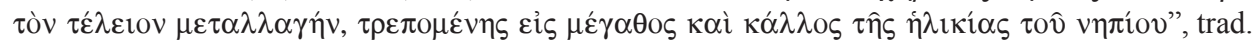
Mejzner - Zorzi, p. 142.

${ }^{74} \mathrm{Cf}$. ibidem III 3, 7-8, dove egli sottolinea che la crescita e l'invecchiamento dell'uomo contraddicono la permanenza della medesima forma ed afferma che è meglio, perciò, parlare della stabilità della carne. Il passo metodiano è conservato nella traduzione paleoslava (manoscritto Q.I. 265 della Biblioteca Nazionale Russa a St. Petersburgo), dove il termine critico è stato reso con la parola овразъ (f. 142). Dal contesto si intende che nell'originale greco dovrebbe esserci proprio il termine

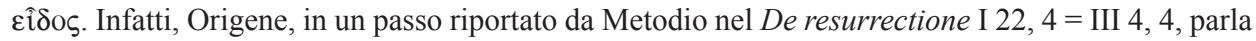

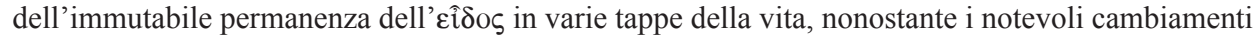

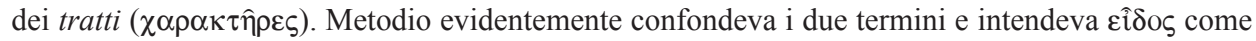
forma esteriore del corpo. 
è molto ridotta. L'autore non parla più del passaggio dalle tende alla terra promessa, e cioè dal paradiso ai cieli, limitandosi ad indicare nella risurrezione, adombrata dalla festa delle tende, la condizione perfetta degli uomini, ivi incluso lo $\sigma \chi \hat{\eta} \mu \alpha$, non chiamato più corruttibile. Tale correzione del proprio pensiero è tanto più comprensibile, se si prende in considerazione il fatto che la questione della forma del corpo risorto, sviluppata in polemica con la concezione origeniana dell' $\varepsilon \hat{i} \delta o \varsigma$, apparteneva alla problematica centrale dell'opera metodiana.

3. Un confronto con alcuni studi moderni. La polemica riguardante l'identità corporea dell'uomo, contenuta nel De resurrectione, è stata analizzata già da alcuni studiosi moderni. Le loro conclusioni non sempre concordano. Perciò sembra utile individuare, sottolineare e valutare le principali variazioni per proporre in seguito delle nuove argomentazioni.

Henri Crouzel, riferendosi allo sforzo argomentativo di Metodio di assicurare l'identità tra il corpo misero e quello glorioso (cf. Fil 3,21) e alla sua polemica con Alessandrino, dice che se egli "avait correctement compris Origène, il aurait vu que ce dernier ne disait pas autre chose"75. Questa osservazione è giusta soltanto in riferimento al complesso pensiero del grande Alessandrino, interessato a spiegare in un nuovo linguaggio, adatto alle esigenze razionalistiche, il dogma della risurrezione. Anche Metodio ammette che Origene non abbia negato questo dogma ${ }^{76}$. Tuttavia la sua posizione critica nei confronti dell'Alessandrino non sembra che sia dovuta soltanto a dei malintesi. Essa risulta come reazione ad alcune scelte terminologiche e concettuali, ritenute non ortodosse o, almeno, pericolose per la dottrina tradizionale. Non bisogna dimenticare, peraltro, che l'attività letteraria di Metodio si svolgeva nel periodo della crescente controversia intorno all'eredità di Origene, causata non soltanto dal contenuto dei suoi scritti, ma anche, o forse soprattutto, dalle interpretazioni deformanti del suo pensiero, avanzate sia dai suoi seguaci che dagli avversari. Gli obiettivi di Metodio erano, quindi, completamente diversi da quegli apologetici di Crouzel. Il vescovo di Olimpo non era interessato a fare un'analisi generale della vasta opera origeniana, ma a confutare singoli concetti, termini e soluzioni ritenuti inconciliabili con la dottrina tradizionale oppure sviluppatisi già verso una direzione eterodossa.

Metodio combatteva in modo particolare il concetto dell' $\varepsilon \hat{i} \delta o \varsigma \sigma \omega \mu \alpha \tau \imath \kappa o ́ v$ di Origene. Per bocca di Memiano, egli tentava di spiegare la persistenza della

${ }^{75}$ Crouzel, Les critiques, p. 700. In seguito (p. 702) Crouzel sottolinea che, secondo Origene, il corpo del Risorto è quel corpo “que Jésus a reçu de Marie: il est pareillement précisé que c'est la chair qu'il a portée. Le changement de la qualité terrestre en qualité éthérée, du moins après l'Ascension, ne fait aucun tort à l'identité d'eidos, donc à celle du corps qu'elle détermine".

${ }^{76}$ Alla conclusione del suo dialogo Metodio riporta un brano tratto dal perduto De resurrectione di Origene, citato anche da Panfilo, che inizia con l'affermazione "a questo corpo spetta risorgere" (III 22, 1). 
materia nel corpo risorto. Nell'analisi di questa polemica, Crouzel sembra attenuare troppo il punto controverso ed identifica quasi le posizioni degli avversari: "Mémianios se contente de montrer que tout ne s'écoule pas, mais que quelque chose demeure: Origène répondrait qu'il est d'accord et que ce qui reste est justement, d'après les arguments de Mémianios, l'eidos corporel. Il y a donc dans cette polémique une grande part de malentendu" "77. Prendendo in considerazione la densità del dibattito dei personaggi di dialogo ed analizzando in maniera dettagliata il contenuto delle loro rispettive argomentazioni, la conclusione dello studioso francese non sembra pertinente.

Le differenze tra le teorie dei due interlocutori sono, invece, giustamente riassunte da Emanuela Prinzivalli: "L' $\varepsilon \hat{i} \delta$ os origeniano è un principio corporeo, che imprime i suoi caratteri alla materia, cioè agisce sul sostrato. Memiano sta parlando proprio di quello che Origene chiamerebbe sostrato: nel caso delle amputazioni è la materia del sostrato che viene a mancare" ${ }^{98}$. Infatti, sebbene Memiano non offra una precisa descrizione degli elementi costitutivi del corpo, la sua posizione si differenzia nettamente da quella di Origene per il fatto che questi elementi appartengono al sostrato materiale e che hanno le qualità fisiche della materia pesante.

È vero, come dimostra Crouzel, che per Origene si tratta del principio di identità corporea, ma è altrettanto vero che tale concetto è segnato da una forte ambiguità sia terminologica che contenutistica. Dalla prospettiva di un autore asiatico degli inizi del IV sec., esso doveva risultare erroneo. L'idea della risurrezione del solo عî́os, cioè di qualcosa di non ben definito e, peraltro, massimamente disincarnato e deformato, non avente né i medesimi elementi materiali né il medesimo assetto delle membra umane, urtava fortemente con la comprensione tradizionale del dogma.

Sintetizzando queste riflessioni si può affermare che per Metodio il concetto

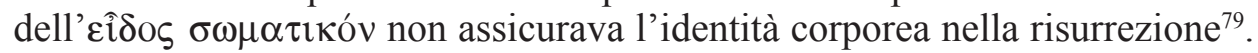
Non è che egli abbia semplicemente frainteso il pensiero dell'Alessandrino.

${ }^{77}$ Crouzel, Les critiques, p. 712.

${ }^{78}$ Prinzivalli, Magister Ecclesiae, p. 110 n. 188. C. Riggi sottolinea l'influsso di Aristotele, La forma del corpo risorto secondo Metodio in Epifanio (Haer. 64), in: Morte e immortalità nella cate-

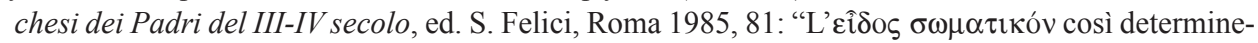
rebbe la materia informe nell'uomo, chiamato da Dio a risorgere nella sua primitiva sostanza. Tale

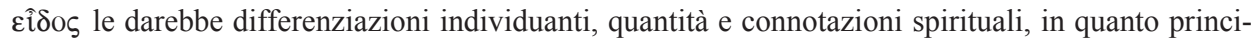
pio dell'ecceità, mediante la materia quantitate signata [...] Metodio ha potuto così cristianizzare Aristotele, contrapponendolo al dualismo platonico che demonizzava la materia, e alla dicotomia origeniana che additava nella materia l'origine del caso e dei mostri".

${ }^{79}$ Eustathius Antiochenus, De engastrimytho contra Origenem XXII 5, afferma che Origene

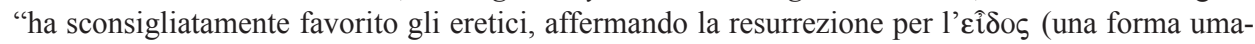
na), ma non per il corpo stesso" (ed. e trad. it. M. Simonetti, in: Origene, Eustazio, Gregorio di Nissa, La maga di Endor, BP 15, Firenze 1989, 179. In questa sintesi fatta da Eustazio è da evidenziare l'opinione che l'Eî่oৎ non assicura l'identità tra il corpo risorto e quello terreno, e che ciò che Origene destina a risorgere non è il corpo stesso. 
Il suo concetto di corpo risorto è proprio diverso: è il corpo terreno, pur trasformato in glorioso, rivestito dell'incorruttibilità e riempito delle azioni dello Spirito Santo, e perciò spirituale ${ }^{80}$, tuttavia restante per sempre di sostanza carnale, cioè composto dagli elementi materiali, senza diventare sottile o aereo.

Una certa minimizzazione dell'importanza dell'identità materiale nel concetto metodiano della risurrezione è presente nelle riflessioni di Katharina Bracht $^{81}$. La categoria della perfezione (Vollkommenheit), fondamentale nel suo studio, spinge la studiosa a sottolineare il ruolo essenziale della potenza, della sapienza e della volontà di Dio nel compimento (Vollendung) escatologico dell'esistenza umana che, come nel caso di tutte le creature, è sempre eine

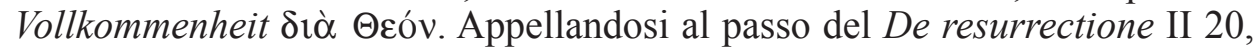
7-9, Bracht accentua che la garanzia dell'identità tra l'uomo terrestre e quello nuovamente creato nella risurrezione risiede soprattutto nella volontà di Dio $^{82}$. Ella critica l'opinione di Herman Josef Vogt, che sottolineava l'importanza primaria dell'identità materiale tra il corpo risorto e quello terreno ${ }^{83}$. Bracht afferma che "die Identität des Menschen liegt nach Methodius viehlmehr in Gott, in seinem Wollen und wiederholten Schöpferhandeln" $"$. La studiosa preferisce parlare della continuità e dell'identità dell'uomo completo (corpo e anima) $)^{85}$ ed osserva che il focalizzarsi metodiano sulla carne, come oggetto della risurrezione, è dovuto alla sua polemica con Origene e con il fronte spiritualizzante, ma che la sua visione antropologica è molto più ampia ${ }^{86}$.

Non si può negare la pertinenza delle considerazioni di Bracht. È vero che, per Metodio, Dio è l'extrema ratio e la garanzia della risurrezione e dell'identità umana nella sua interezza. Bisogna sottolineare, tuttavia, che la questione centrale del dibattito e, quindi, della sua argomentazione, consiste indubbiamente nell'assicurazione dell'identità materiale e formale tra il corpo terreno e quello risorto. Per Metodio la causa materiale è essenziale nel suo concetto di identità corporea: il corpo è identico non per una decisione arbitraria di Dio, ma perché Egli lo ricostruisce dagli stessi elementi. Le analisi di Bracht,

\footnotetext{
${ }^{80} \mathrm{Cf}$. Methodius, De resurrectione III 16, 9.
}

${ }^{81} \mathrm{Cf}$. K. Bracht, Vollkommenheit und Vollendung. Zur Anthropologie des Methodius von Olympus, Studien und Texte zu Antike und Christentum 2, Tübingen 1999, 302-321.

${ }^{82}$ Cf. ibidem, p. 318: "Für die Neuschaffung des menschlichen Leibes in der Auferstehung sind nicht allein Gottes Kraft und Fähigkeit von Bedeutung, sondern auch Gottes Wollen - und darin liegt die Garantie für die Identität des erstgeschaffenen irdischen und des neugeschaffenen Menschen.

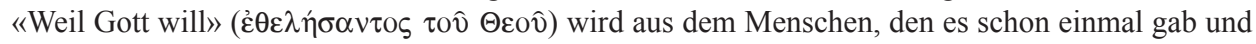
der schon einmal von Gott als ein vollkommener Mensch geschaffen worden war, wiederum dasselbe, nämlich wiederum ein Mensch".

${ }^{83}$ Cf. H.-J. Vogt, Warum wurde Origenes zum Häretiker erklärt? Kirchliche VergangenheitsBewältigung in der Vergangenheit, in Origeniana quarta, Innsbruck - Wien 1987, 89: "die Identität der Person in die rein materielle Identität des Leibes verlegt".

${ }^{84}$ Bracht, Vollkommenheit und Vollendung, p. 318, n. 44.

${ }^{85}$ Cf. ibidem, p. 304.

${ }^{86}$ Cf. ibidem, p. 309. 
concentrandosi sulla prospettiva della perfezione, diminuiscono l'importanza dell'identità materiale nell'argomentazione di Metodio, invertendo così gli obiettivi della sua opera.

Metodio, strenuo difensore del dogma della risurrezione della carne, ha contrapposto ad Origene e ad altri avversari, una visione precisa del corpo risorto, la cui identità è garantita sia nella materia (dagli elementi costitutivi) che nella

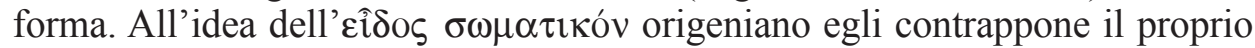
concetto dell'identità tra il corpo risorto e quello terreno. Per confermarlo, egli adopera una specifica esegesi di certi passi scritturistici e rievoca alcuni esempi presi dal mondo della natura. Secondo Metodio, alla fine dei tempi, Dio onnipotente attraverso il Cristo che è causa e modello della risurrezione, farà risorgere tutti gli uomini, ricomponendo i loro propri corpi dagli elementi costitutivi. I corpi risorti, pur materiali, avranno delle caratteristiche nuove di immortalità, incorruttibilità e impassibilità e saranno spirituali, cioè sintonizzati all'azione prepotente dello Spirito Santo che dimorerà in essi per tutta l'eternità.

\section{AN APOLOGY OF THE IDENTITY OF THE RESURRECTED BODY IN RESPECT OF THE EARTHLY ONE IN METHODIUS OF OLYMPUS' DE RESURRECTIONE}

\section{(Summary)}

Methodius' Discourse on the Resurrection (De resurrectione) is an extensive and profound presentation of the ecclesiastic understanding of this dogma in the context of the dissent towards certain opinions of Origen, and Platonic currents in Christianity. At the onset of the IV century, one of the central theological problems lay in demonstrating the rationality of the conceptualisation of "the resurrection of the flesh", as well as a detailed explanation of its essence. The aim of this article is to indicate and describe those elements acknowledged by Methodius as necessary and constitutive for the orthodox understanding of the dogma. A further aim is an analysis of the application of his argumentations, focused on evidencing the identity of the resurrected body in respect of the earthly one, in both matter and form. The article also attempts to understand the essential motivations which induced the bishop of Olympus to address the very complex issue of resurrection during the Diocletianic persecutions. In light of earlier studies, an evaluation of the argumentations of the author of De resurrectione is proposed, in the context of the doctrinal debates of his times. 
OBRONA TOŻSAMOŚCI CIAŁA ZMARTWYCHWSTAŁEGO Z ZIEMSKIM W DE RESURRECTIONE METODEGO Z OLIMPU

\section{(Streszczenie)}

Dialog Metodego O zmartwychwstaniu (De resurrectione) jest obszernym i pogłębionym przedstawieniem kościelnego rozumienia tego dogmatu w kontekście polemiki z niektórymi poglądami Orygenesa i platonizujących nurtów w chrześcijaństwie. W początkach IV w., jednym z kluczowych problemów teologicznych było wykazanie racjonalności formuły „zmartwychwstanie ciała” oraz szczegółowe wyjaśnienie jej treści. Celem artykułu jest wskazanie i opisanie elementów, uznanych przez Metodego za konieczne i konstytutywne dla prawowiernego rozumienia dogmatu oraz analiza zastosowanej przez niego argumentacji, ukierunkowanej na wykazanie tożsamości ciała zmartwychwstałego z ziemskim, tak co do materii, jak i formy. W artykule podjęta też została próba zrozumienia zasadniczych motywacji, które w okresie prześladowań Dioklecjańskich skłoniły biskupa Olimpu do zajęcia się w sposób tak kompleksowy kwestią zmartwychwstania. W świetle dotychczasowych studiów zaproponowana też została ocena argumentacji autora De resurrectione w kontekście doktrynalnej polemiki w jego czasach.

Key words: Methodius of Olympus, Origen, resurrection, identity of the body, the form of the body, constitutive elements.

Parole chiave: Metodio di Olimpo, Origene, risurrezione, identità del corpo, forma corporea, elementi constitutive.

Słowa kluczowe: Metody z Olimpu, Orygenes, zmartwychwstanie, tożsamość ciała, forma ciała, elementy konstytutywne.

\section{BIBLIOGRAFIA}

Fonti

Acta Phileae, ed. E. Bastiaensen, in:, Atti e passioni dei martiri, Milano 1987, 280-337.

Eustathius Antiochenus, De engastrimytho contra Origenem, ed. e trad. it. M. Simonetti, in: Origene, Eustazio, Gregorio di Nissa, La maga di Endor, BP 15, Firenze 1989, 94-207.

Ireneaus, Adversus haereses V, ed. A. Rousseau - L. Doutreleau, SCh 153, Paris 1969, trad. it. E. Bellini, in: Ireneo di Lione, Contro le eresie e gli altri scritti, Milano 1997.

Iustinus, Dialogus cum Tryphone, ed. M. Marcovich, PTS 47, Berlin 1997, trad. it. G. Visonà: San Giustino, Dialogo con Trifone, Milano 1988.

Martyrium Lugdunensium, ed. A. Bastiaensen, in: Atti e passioni dei martiri, Milano 1987, 59-95.

Methodius, De martyribus, ed. G.N. Bonwetsch, GCS 27, Leipzig 1917, 520.

Methodius, De resurrectione, ed. G.N. Bonwetsch, GCS 27, Leipzig 1917, 219-424, trad. it. M. Mejzner - B. Zorzi: Metodio di Olimpo, La risurrezione, Collana di Testi Patristici 216, Roma 2010. 
Methodius, Symposium, ed. H.A. Musurillo - V.-H. Debidour, SCh 95, Paris 1963, trad. it. N. Antoniono: Metodio d'Olimpo, La verginità, Collana di Testi Patristici 152, Roma 2000.

Origenes, Contra Celsum V-VI, ed. M. Borret, SCh 147, Paris 1969.

Origenes, Homiliae in Exodum, ed. M. Borret, SCh 321, Paris 1985.

Origenes, Homiliae in Numeros, ed. W.A. Baehrens, GCS 7, Leipzig 1921, 3-285.

Pнотіus, Bibliotheca, ed. R. Henry - J. Schamp: Photius: Bibliothèque, I-IX, Paris 1962-1991.

Pseudo-Iustinus, De resurrectione, ed. e trad. it. A. D’Anna: Pseudo-Giustino, Sulla resurrezione. Discorso cristiano del II secolo, Brescia 2001.

Tertullianus, Adversus Marcionem III, ed. R. Braun, SCh 399, Paris 1994, trad. it. C. Moreschini, in: Tertulliano, Opere scelte, Torino 1999, 109-519.

Tertullianus, De resurrectione mortuorum, ed. J.G. Borleffs, CCL 2, Turnholti 1954, 921-1012, trad. it. C. Moreschini, in: Tertulliano, Opere scelte, Torino 1999, 523-638.

Studi

Biamonti A., L'escatologia di Metodio d'Olimpo, "Rivista Trimestrale di Studi Filosofici e Religiosi" 4 (1923) 182-202.

Becker J., La risurrezione dei morti nel cristianesimo primitivo, Studi Biblici 97, Brescia 1991.

Bonwetsch G.N., Die Theologie des Methodius von Olympus, Abhandlungen der Königlichen Gesellschaft der Wissenschaften zu Gottingen, Philologisch-Historische Klasse, Neue Folge Band VII/1, Berlin 1903.

Bracht K., Vollkommenheit und Vollendung. Zur Anthropologie des Methodius von Olympus, Studien und Texte zu Antike und Christentum 2, Tübingen 1999.

Crouzel H., Les critiques adressées par Méthode d'Olympe et ses contemporains à la doctrine origenienne du corps ressuscité, “Gregorianum” 53 (1972) 679-716.

DaniÉLou J., La risurrezione, Torino 1970.

D’Anna A., Studio critico, in: Pseudo-Giustino, Sulla resurrezione. Discorso cristiano del II secolo, Brescia 2001, 57-287.

Dorival G., Origène et la résurrection de la chair, in: Origeniana quarta. Die Referate des 4. Internationalen Origeneskongresses, Innsbruck, 2-6 September 1985, ed. L. Lies, Innsbruck - Wien 1987, 291-321.

Kortekans G., Prefazione: Atti di Filea, in: Atti e passioni dei martiri, ed. A. Bastiaensen, Milano 1987, 249-270.

Lona H.E., Über die Auferstehung des Fleisches. Studien zur frühchristlichen Eschatologie, Berlin - New York 1993.

LoRenz R., Eine Pierius-Memoria in Alexandrien, ZKG 99 (1988) 87-92.

Marcheselli-Casale C., Risorgeremo, ma come? Resurrezione dei corpi, degli spiriti o dell'uomo? Per un contributo allo studio della speculazione apocalittica in epoca greco-romana: II a.C.-II d.C., Bologna 1988.

Mejzner M., Escatologia di Metodio di Olimpo, SEA 124, Roma - Ząbki 2011.

MeJzNer M., L'eidos e l'ochèma. La critica al concetto origeniano di risurrezione nel contesto dell'escatologia intermedia nel "De resurrectione" di Metodio di Olimpo, in: Origeniana Decima: Origen as Writer. Papers of the $10^{\text {th }}$ International Origen Congress, Krakow: 31.08 - 04.09.2009, ed. S. Kaczmarek - H. Pietras, Bibliotheca Ephemeridum Theologicarum Lovaniensium 244, Leuven - Paris - Walpole 2011, 907-918. 
MeJzner M., Methodius: millenarist or anti-millenarist?, in: Methodius of Olympus. State of the art and new perspectives, ed. K. Bracht, TU, Berlin 2017 [in via di pubblicazione].

Patterson L.G., Methodius'Millenarianism, StPatr 24 (1993) 306-315.

Prinzivalli E., L'esegesi biblica di Metodio di Olimpo, SEA 21, Roma 1985.

Prinzivalli E., La risurrezione nei Padri, in: Dizionario di Spiritualità Biblico-Patristica 45, Roma 2007, 169-288.

Prinzivalli E., Magister Ecclesiae. Il dibattito su Origene fra III e IV secolo, SEA 82, Roma 2002.

Riggi C., La forma del corpo risorto secondo Metodio in Epifanio (Haer. 64), in: Morte e immortalità nella catechesi dei Padri del III-IV secolo, ed. S. Felici, Roma 1985, 75-92.

Simonetti M., Alcune osservazioni sull'interpretazione origeniana di Gn 2,7 e 3,21, "Aevum" 36 (1962) 370-381.

Szram M., Ciało zmartwychwstałe w myśli patrystycznej przełomu II i III wieku, Lublin 2010.

Vogt H.-J., Warum wurde Origenes zum Häretiker erklärt? Kirchliche VergangenheitsBewältigung in der Vergangenheit, in: Origeniana quarta. Die Referate des 4. Internationalen Origeneskongresses, Innsbruck, 2-6 September 1985, ed. L. Lies, Innsbruck - Wien 1987, 78-99.

Wysocki M., Eschatologia okresu prześladowań na podstawie pism Tertuliana i Cypriana, Lublin 2010. 